\title{
2015 Awards in the Journal of Plant Research
}

\author{
Ikuo Nishida ${ }^{1}$
}

Published online: 8 August 2015

(C) The Botanical Society of Japan and Springer Japan 2015

Each year, the Botanical Society of Japan (BSJ) honors excellence in publications of the Journal of Plant Research (JPR) through the Best Paper Awards and the MostCited Paper Award. We are proud to announce this year's recipients.

\section{Best Paper Awards}

Masakazu Tomiyama, Shin-ichiro Inoue, Tomo Tsuzuki, Midori Soda, Sayuri Morimoto, Yukiko Okigaki, Takaya Ohishi, Koji Takahashi, and Toshinori Kinoshita in the Division of Biological Science in the Graduate School of Science at Nagoya University and Nobuyoshi Mochizuki in the Department of Botany in the Graduate School of Science at Kyoto University have found a positive link between chlorophyll biosynthesis and ABA signaling in the control of stomatal aperture (Tomiyama et al. 2014). They isolated a low temperature with open-stomata 1 (lost1) mutant that showed reduced leaf temperature due to stomatal malfunction using infrared thermography. The lost1 mutant showed ABA-insensitivity and had a missense mutation in the Mg-chelatase I subunit 1 (CHLI1) gene, which encodes a subunit of the Mg-chelatase complex. They conclude that together with similar phenotypes of other mutants in the chlorophyll biosynthetic pathway, the chlorophyll synthesis enzymes specifically affect ABA signaling in the control of stomatal aperture. Interestingly,

Ikuo Nishida

jpreic@gr.saitama-u.ac.jp

Division of Life Science, Graduate School of Science and Engineering, Saitama University, 255 Shimo-Okubo, Sakura-Ku, Saitama 338-8570, Japan the mutants in chlorophyll biosynthesis have no effect on ABA-induced gene expression. Therefore, further research may reveal a novel ABA-signaling pathway in the control of stomatal aperture.

Suzana Pampurova, Katrien Verschooten, Nelson Avonce, Patrick Van Dijck in the Department of Molecular Microbiology at KU Leuven, Belgium, have found an interesting plant-microbe cooperation in the biosynthesis of trehalose in the desiccation-tolerant plant Selaginella lepidophylla (Pampurova et al. 2014). This plant accumulates very high levels of trehalose in both the hydrated and dehydrated state, but the mechanism of trehalose biosynthesis remains unexplained. By cDNA cloning of an ortholog of trehalose-6-phosphate phosphatase, the final enzyme of the trehalose biosynthetic pathway, they found that the isolated clone is derived from microbial rather than plant origin. Because S. lepidophylla has been known to have endophytes, it is most likely that S. lepidophylla utilizes microbes to accumulate trehalose. It remains unclear, however, why trehalose is accumulated in S. lepidophylla, because trehalose levels are not correlated with desiccation tolerance ability of this plant.

\section{Most-Cited Paper Award}

Masayuki Muramatsu and Yukako Hihara at Saitama University published a review article in January 2012 on physiological and genetic acclimation of cyanobacteria to high light conditions (Muramatsu and Hihara 2012). To acclimate to a changing light environment, photosynthetic organisms have evolved a balancing mechanism between light energy harvesting and light energy utilization. The mechanism is based on cellular metabolic regulation and genetic control. In this review, Muramatsu and Hihara 
focused on the mechanism of photosynthetic gene expression in response to high light exposure in cyanobacteria. This article has been cited 23 times since 2012, excluding the authors' own citation.

\section{Our 5-year plan for improvement is approved}

Our 2015-2019 plan to increase the global credibility of JPR as an international journal of basic plant sciences has been accepted by the Japan Society for the Promotion of Science (JSPS KAKENHI Grant Number 15HP1002). With this support, we are encouraging all of you to propose a topic for an international JPR symposium at the BSJ annual meeting and submit symposium papers to JPR. The impact factor of JPR in 2014 has been announced as 1.823 . You are cordially invited to continue submitting excellent papers to JPR.

\section{From the editorial office}

We have revised our "Instructions to Authors" (30 October 2014), which include some important statements of publication ethics.
We have been working with a new Editor, Hajime Ikeda, since 9 March and a new Editorial Board member, Satoshi Koi, since 1 April, both in the field of taxonomy/phylogenetics/evolution biology.

Ikuo Nishida

Editor-in-Chief, Journal of Plant Research

\section{References}

Muramatsu M, Hihara Y (2012) Acclimation to high light conditions in cyanobacteria: from gene expression to physiological responses. J Plant Res 125:11-39

Pampurova S, Verschooten K, Avonce N, Van Dijck P (2014) Functional screening of a cDNA library from the desiccation-tolerant plant Selaginella lepidophylla in yeast mutants identifies trehalose biosynthesis genes of plant and microbial origin. J Plant Res 127:803-813

Tomiyama M, Inoue S, Tsuzuki T, Soda M, Morimoto S, Okigaki Y, Ohishi T, Mochizuki N, Takahashi K, Kinoshita T (2014) Mgchelatase I subunit 1 and Mg-protoporphyrin IX methyltransferase affect the stomatal aperture in Arabidopsis thaliana. J Plant Res 127:553-563 